Proceedings of the 45th International School and Conference on the Physics of Semiconductors "Jaszowiec" 2016, Szczyrk

\title{
Hardening of $(\mathrm{Pb}, \mathrm{Cd})$ Te Crystal Lattice with an Increasing CdTe Content in the Solid Solution
}

\author{
R. Kuna ${ }^{a}$, S. AdAmiak ${ }^{b}, \mathrm{~S} . \mathrm{Petit}^{c}, \mathrm{P} . \mathrm{BARONi}^{c}, \mathrm{~K} . \mathrm{GaS}^{a, d}, \mathrm{R} . \mathrm{MinikAYeV}^{a}$, \\ A. SzCZERBAKOW ${ }^{a}$, J. ŁAŻEWSKi ${ }^{e}$ AND W. SzUSZKIEWICz ${ }^{b, a *}$ \\ ${ }^{a}$ Institute of Physics, Polish Academy of Sciences, Aleja Lotnikow 32/46, PL-02668 Warsaw, Poland \\ ${ }^{b}$ Faculty of Mathematics and Natural Sciences, University of Rzeszów, S. Pigonia 1, PL-35310 Rzeszów, Poland \\ ${ }^{c}$ Laboratoire Léon Brillouin, CEA-CNRS, CEA Saclay, F-91191 Gif-sur-Yvette Cedex, France \\ ${ }^{d}$ Institute of Experimental Physics, Faculty of Physics and Astronomy, University of Wrocław, \\ Pl. M. Borna 9, PL-50204 Wrocław, Poland \\ ${ }^{e}$ Institute of Nuclear Physics, Polish Academy of Sciences, E. Radzikowskiego 152, PL-31342, Kraków, Poland
}

\begin{abstract}
Single crystals of the $(\mathrm{Pb}, \mathrm{Cd}) \mathrm{Te}$ solid solution with $\mathrm{CdTe}$ content up to $9 \%$ were grown by self-selecting vapour growth method and investigated by powder X-ray diffraction, inelastic neutron scattering, and nanoindentation measurements. The analysis of the linear part of the LA phonon dispersion, determined by the inelastic neutron scattering demonstrated an increase of the sound velocity (thus the hardening of the crystal lattice) with an increase of CdTe content in the solid solution. An important increase of microhardness value for $(\mathrm{Pb}, \mathrm{Cd}) \mathrm{Te}$ was directly confirmed by results of nanoindentation measurements performed for a few samples with various chemical composition.
\end{abstract}

DOI: $10.12693 /$ APhysPolA.130.1245

PACS/topics: 62.20.-x, 63.20.D-

\section{Introduction}

Lead telluride is one from the lead chalcogenides, which has found much usefulness in the field of thermoelectricity and infrared detection. The first papers dedicated to the hardness determination for this compound appeared almost fifty years ago $[1,2]$. In single $n$-type PbTe bulk crystal grown by the Bridgman method the microhardness is almost a constant value of $H \approx 300 \mathrm{MPa}$ for various electron concentration whereas in $p$-type material this value strongly increases with the hole concentration and can be as high as $H \approx 700 \mathrm{MPa}[3,4]$. Due to the relatively low $\mathrm{PbTe}$ microhardness value a layer of this compound is so soft that it can be scratched easily. As a consequence various devices consisting of PbTe layers are not robust enough to withstand the damage originating from standard fabrication processing. In order to avoid this problem more robust materials are chosen typically as an outermost layer in selected devices. However, another possibility is to replace the $\mathrm{PbTe}$ by a solid solution containing some amount of other compound. This idea is not a new one. A softening of PbTe by alloying of this compound with small amount of SnTe (up to 3\%) was demonstrated in [1], solid ( $\mathrm{Pb}, \mathrm{Ca}) \mathrm{Te}$ solution containing up to $6 \%$ of CaTe was the topic of recent studies [5]. Up to our knowledge in the case of thin PbTe-based layers the alloying was limited to the GeTe only with its content not higher than $22 \%[6,7]$. A hardening of $\mathrm{PbTe}$ crystals by alloying it with CdTe was suggested a long

\footnotetext{
*corresponding author; e-mail: szusz@ifpan.edu.pl
}

time ago [3], but due to lack of access to the $(\mathrm{Pb}, \mathrm{Cd}) \mathrm{Te}$ solid solutions with good structure quality these studies were limited to samples with one nominal CdTe content equal at least to $3 \%$ in the past.

The successful growth of big, high quality single $(\mathrm{Pb}, \mathrm{Cd}) \mathrm{Te}$ crystals with $\mathrm{CdTe}$ content as high as $12 \%$ by self-selecting vapour growth (SSVG) method changed this situation $[8,9]$. Some results obtained using these crystals can be found in $[10,11]$. The aim of present studies of the $(\mathrm{Pb}, \mathrm{Cd}) \mathrm{Te}$ solid solution was to analyse in a more systematic manner the crystal lattice hardening resulting from an increasing doping. Both a non-direct method (a modification of the sound velocity with lattice hardening demonstrated by studies of the linear part of LA phonon dispersion by an inelastic neutron scattering (INS) technique) and the direct one (the nanoindentation measurements performed on oriented single crystals) were selected for such purpose.

\section{Experimental and calculations details}

The single $(\mathrm{Pb}, \mathrm{Cd}) \mathrm{Te}$ crystals containing from 0 to $9 \%$ of the CdTe were grown at the Institute of Physics, PAS, by the self-selecting vapour growth (SSVG) method. All samples were $p$-type crystals with relatively high hole concentration [9]. The structure characterization of these crystals was performed first by powder X-ray diffraction at the same laboratory using $\mathrm{Cu} K_{\alpha_{1}}$ radiation and next (for the single crystals) by neutron diffraction using G2.4 neutron spectrometer installed on the cold neutron source at Laboratoire Léon Brillouin in France. The same spectrometer served for the inelastic neutron scattering (INS) measurements at room temperature on 
selected samples, applied for the determination of linear part of longitudinal acoustic (LA) phonon dispersion along the [001] high symmetry direction in the vicinity of the Brillouin zone centre. The microhardness and the Young modulus were determined for natural, (001)oriented faces of a few single crystals by the nanoindentation method. For the measurements mentioned above an ultra nanohardness tester CSM with the following parameters was used: maximum load $1 \mathrm{mN}$, linear change of the load during application or removal of the load $0.033 \mathrm{mN} / \mathrm{s}$, application time of the maximum load $30 \mathrm{~s}$.

All first-principles calculations were made in the frame of density functional theory (DFT) as implemented in VASP package [12]. For detailed setup see Sect. 2 in Ref. [13]. The high accuracy of this approach for both structural as well as dynamical properties of solids was confirmed in many papers (see, e.g. [14]). The elastic properties of ideal $\mathrm{PbTe}$ crystals were determined from method based on the stress-strain relationship described in Refs. $[15,16]$. The discussion of the results obtained in Ref. [17] against wrong experimental findings clearly illustrates capabilities and the power of this method.

\section{Results and discussion}

The structure analysis of all investigated single crystals demonstrated their good quality and excluded possible presence of other phases or precipitates. Next, the LA phonon dispersion was determined along [001] direction for selected solid solutions by the INS. Figure 1 shows the experimental points (squares) corresponding to the sample containing $5 \%$ of CdTe. This figure illustrated

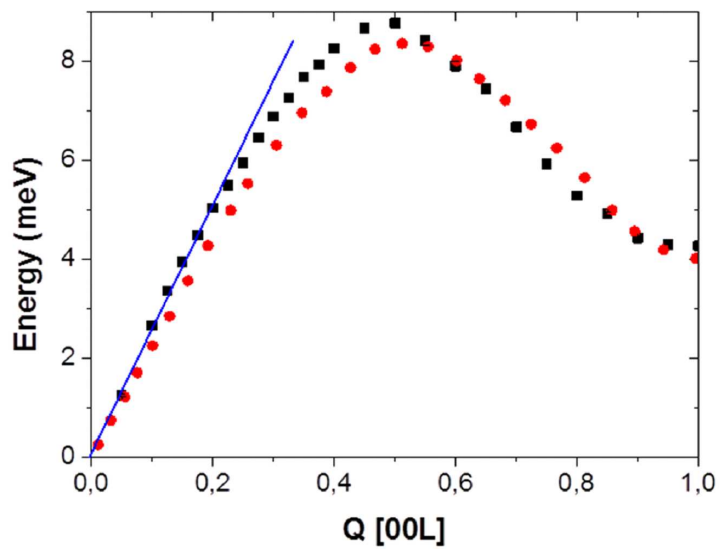

Fig. 1. Comparison of LA phonon dispersion along [001] direction measured at room temperature by INS for (Pb,Cd)Te with $5 \%$ of CdTe (squares) and calculated for $\mathrm{PbTe}$ (circles). The linear part of the first dispersion is indicated (details in the text).

the method of the estimation of the velocity of sound applied in the present paper. From the slope of the tangent line to the LA phonon dispersion in the vicinity of the Brillouin zone centre (an area of a linear dispersion) the velocity of sound was determined. In order to demonstrate the change of this slope with an increasing CdTe content in the solid solution, the theoretical LA phonon dispersion of PbTe is shown for the comparison. The theoretical dispersion (circles) obtained using ab initio calculations and the supercell method described very well most of the experimental points determined for this compound. These two dispersions looked very similar but using the INS technique it was possible without any doubt to demonstrate small differences between them. From the analysis of modification of linear part of the LA phonon dispersion, determined by the INS, a noticeable increase of the sound velocity with an increasing CdTe content in $(\mathrm{Pb}, \mathrm{Cd}) \mathrm{Te}$ solid solution was found.

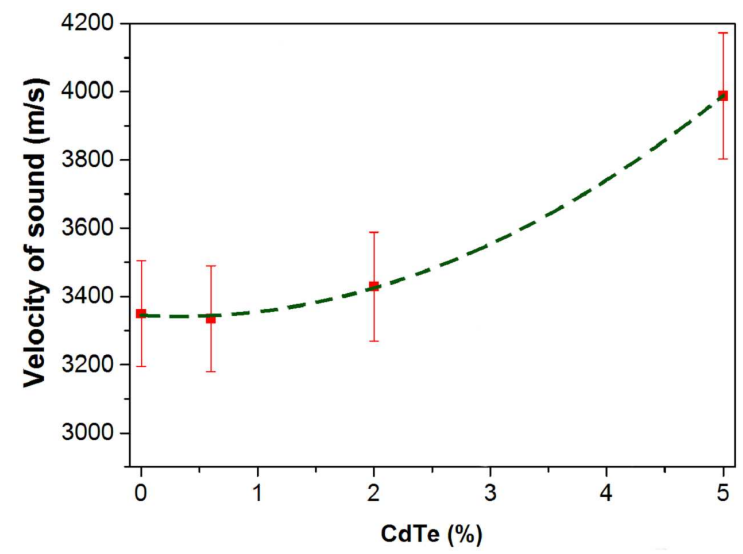

Fig. 2. Composition dependence of the sound velocity in $(\mathrm{Pb}, \mathrm{Cd}) \mathrm{Te}$ solid solution resulting from the INS measurements performed at room temperature. Dashed line is a guide to the eye.

This result is shown in Fig. 2. The numerical values are subjected to noticeable experimental error equal at the very least to $5 \%$ (resulting mostly from a limited accuracy of very low values of LA phonon mode frequencies in the area of interest), nevertheless the general trend is well seen in this figure. As it was shown in Ref. [15] elastic properties can be more accurately determined from stress-strain relation than from acoustic phonon dispersion. The theoretical value of sound velocity in $\mathrm{PbTe}$ along [100] direction obtained on this way is equal to $3699.8 \mathrm{~ms}^{-1}$. An increase of the sound velocity clearly indicates the crystal lattice hardening and cannot be explained simply by a small modification of crystal density resulting from partial replacement of $\mathrm{Pb}$ by $\mathrm{Cd}$. It could be mentioned that the modification of the LA phonon dispersion with changing composition of the solid solution is not only limited to the change of the tangent line slope described above. The small shift in both the energy and the wave vector value corresponding to the LA phonon maximum is well seen in Fig. 1.

More direct experimental data determined by nanoindentation measurements confirmed the solid solution lattice hardening with an increase of CdTe content. Figure 3 illustrates resulting from this phenomenon decrease of the nanoindentation depth with an increase of alloying. 
The average values and standard deviations of the microhardness $H$ and the Young modulus $E$ were extracted from the determined load-displacement curves. They are presented in Table I. The theoretical $E$ value for PbTe obtained from calculated elastic tensor reads 70.0 GPa. The results of present measurements demonstrate a significant increase of the microhardness value observed in $(\mathrm{Pb}, \mathrm{Cd}) \mathrm{Te}$ solid solution in comparison to that of PbTe. The change of the microhardness value is not accompanied by a clear modification of the Young modulus, which seems to be almost independent of the chemical composition in the analysed range of the CdTe content in the solid solution.

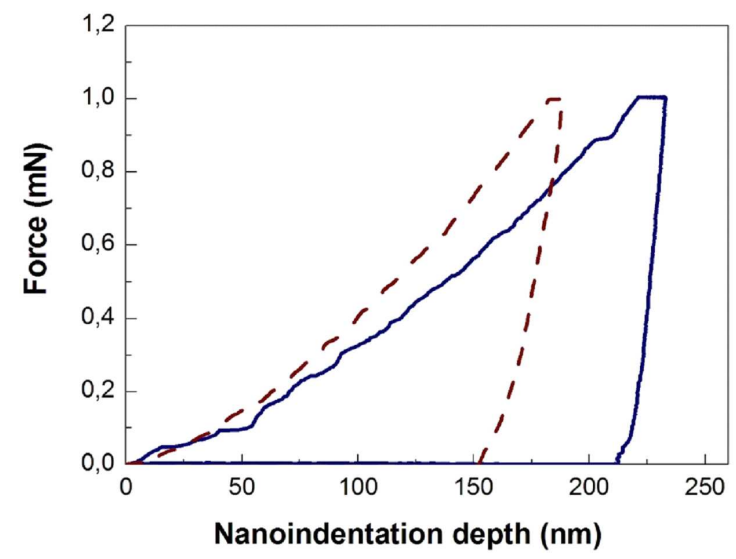

Fig. 3. Load-depth curves determined by nanoindentation for $\mathrm{PbTe}$ and $(\mathrm{Pb}, \mathrm{Cd}) \mathrm{Te}$ solid solution containing $2 \%$ of CdTe (solid line - PbTe, dashed line $(\mathrm{Pb}, \mathrm{Cd}) \mathrm{Te})$.

TABLE I

The microhardness and the Young modulus values for analyzed single crystals of $(\mathrm{Pb}, \mathrm{Cd}) \mathrm{Te}$ solid solution.

\begin{tabular}{c|c|c}
\hline \hline Composition & \multicolumn{2}{|c}{ Mechanical properties } \\
\hline CdTe & $\begin{array}{c}\text { microhardness } \\
{[\mathrm{MPa}]}\end{array}$ & $\begin{array}{c}\text { Young modulus } \\
{[\mathrm{GPa}]}\end{array}$ \\
\hline$\%]$ & $894 \pm 30$ & $72 \pm 4$ \\
2 & $1300 \pm 47$ & $73 \pm 6$ \\
5 & $1431 \pm 41$ & $52 \pm 3$ \\
9 & $1640 \pm 60$ & $72 \pm 4$
\end{tabular}

\section{Conclusions}

An important $(\mathrm{Pb}, \mathrm{Cd}) \mathrm{Te}$ crystal lattice hardening with an increasing $\mathrm{CdTe}$ content in the solid solution was demonstrated by two independent experimental methods: the INS and the nanoindentation. The same time the Young modulus value within an experimental error remained constant and equal to that of $\mathrm{PbTe}$ sample.

\section{Acknowledgments}

This work was supported in part by National Science Centre (Poland) through grant UMO2014/13/B/ST3/04393 and by the EU 7th Framework Programme under the project REGPOT-CT-20133160014 (EAgLE) and the NMI3-II Grant no. 283883.

\section{References}

[1] M.S. Ablova, M.N. Vinogradova, M.N. Karklina, Sov. Phys. Solid State 10, 2089 (1969).

[2] M.S. Ablova, M.N. Karklina, M.N. Vinogradova, Sov. Phys. Solid State 11, 2089 (1970).

[3] A.J. Crocker, M. Wilson, J. Mater. Sci. 13, 833 (1978).

[4] Y. Gelbstein, Z. Dashevsky, M.P. Dariel, J. Appl. Phys. 104, 033702 (2008).

[5] A. Schmitz, J. De Boor, K. Mull, E. Muller, J. Mater. Sci. 51, 6933 (2016).

[6] B. Li, P. Xie, S. Zhang, D. Liu, J. Mater. Sci. 46, 4000 (2011).

[7] B. Li, P. Xie, S. Zhang, D. Liu, Adv. Mater. Res. 455-456, 8 (2012).

[8] A. Szczerbakow, K. Durose, Prog. Cryst. Growth Charact. Mater. 51, 81 (2005).

[9] M. Szot, A. Szczerbakow, K. Dybko, L. Kowalczyk, E. Smajek, V. Domukhovski, E. Łusakowska, P. Dziawa, A. Mycielski, T. Story, M. Bukała, M. Galicka, P. Sankowski, R. Buczko, P. Kacman, Acta Phys. Pol. A 116, 959 (2009).

[10] B.A. Orlowski, A. Szczerbakow, B.J. Kowalski, M.A. Pietrzyk, K. Gas, M. Szot, W. Szuszkiewicz, V. Domukhovski, A. Reszka, S. Mickevicius, R.L. Johnson, S. Thiess, W. Drube, J. Electr. Spec. Rel. Phenom. 184, 199 (2011).

[11] R. Minikayev, E. Dynowska, E. Kaminska, A. Szczerbakow, D. Trots, T. Story, W. Suszkiewicz, Acta Phys. Pol. A 119, 699 (2011).

[12] G. Kresse, J. Furthmüller, Comput. Mater. Sci. 6 , 15 (1996); Phys. Rev. B 54, 11169 (1996).

[13] R. Kuna, R. Minikayev, M. Trzyna, K. Gas, A. Bosak, A. Szczerbakow, S. Petit, J. Łażewski, W. Szuszkiewicz, Acta Phys. Pol. A 130, 1251 (2016).

[14] J. Łażewski, P.T. Jochym, K. Parlinski, P. Piekarz, J. Mol. Struct. 596, 3 (2001).

[15] P.T. Jochym, K. Parlinski, M. Sternik, Eur. Phys. J. B 10, 9 (1999).

[16] P.T. Jochym, K. Parlinski, Eur. Phys. J. B 15, 265 (2000).

[17] J. Łażewski, H. Neumann, K. Parlinski, Phys. Rev. B 70, 195206 (2004). 